\title{
Frequent somatic demethylation of RAPGEF1/C3G intronic sequences in gastrointestinal and gynecological cancer
}

\author{
JOHANNA SAMUELSSON $^{1,2}$, SERGIO ALONSO ${ }^{2}$, TATIANA RUIZ-LARROYA ${ }^{2,3}$, \\ TAK HONG CHEUNG ${ }^{4}$, YICK FU WONG ${ }^{4}$ and MANUEL PERUCHO ${ }^{2,3,5}$
}

\author{
${ }^{1}$ Graduate School of Biomedical Sciences, Sanford-Burnham Medical Research Institute (SBMRI), 10901 North Torrey \\ Pines Road, La Jolla, CA 92037, USA; ${ }^{2}$ Institute of Predictive and Personalized Medicine of Cancer (IMPPC), \\ Carretera de Can Ruti S/N, 08916 Badalona, Barcelona, Spain; ${ }^{3}$ Sanford-Burnham Medical Research Institute (SBMRI), \\ 10901 North Torrey Pines Road, La Jolla, CA 92037, USA; ${ }^{4}$ Department of Obstetrics and Gynaecology, \\ The Chinese University of Hong Kong, Prince of Wales Hospital, Shatin, N.T., Hong Kong, P.R. China \\ ${ }^{5}$ Catalan Institution for Research and Advanced Studies (ICREA), Pg. Lluís Companys 23, 08010 Barcelona, Spain
}

Received January 4, 2011; Accepted February 4, 2011

DOI: $10.3892 /$ ijo.2011.972

\begin{abstract}
RAPGEF1 (also known as C3G and GRF2) is a guanine nucleotide exchange factor that releases GDP from the inactive Rap1 protein, facilitating its subsequent activation by the binding of GTP. Rap1 plays regulatory roles in proliferation, differentiation and apoptosis. Amplification and overexpression of RAPGEF1 have been found in small cell lung cancers, suggesting an oncogenic role. In contrast, hypermethylation of a promoter CpG island (CGI-A) of RAPGEF1 has been reported in squamous cervical tumors, suggesting an anti-oncogenic role in these gynecological cancers. In our studies of DNA methylation alterations in gastrointestinal cancer we found somatic demethylation of a relaxed-criterion $\mathrm{CpG}$ island (CGI-B) located in the first intron of RAPGEF1 in $40 \%$ of colon cancers and $8 \%$ of gastric cancers relative to their matching normal tissues that were always methylated. We also found somatic demethylation in $47 \%$ of squamous cervical carcinomas as well as $33 \%$ of ovarian cancers. This somatic change in methylation, however, did not extend to the strict-criterion $\mathrm{CpG}$ island located in the promoter region (CGI-A) that was unmethylated in all normal and tumor tissues analyzed. Thus, promoter hypermethylation of RAPGEF1 seems insignificant in colorectal, cervical and ovarian cancers.
\end{abstract}

Correspondence to: Dr Manuel Perucho, Institute of Predictive and Personalized Medicine of Cancer (IMPPC), Carretera de Can Ruti S/N, 08916 Badalona, Barcelona, Spain

E-mail: mperucho@imppc.org

Abbreviations: MS-AFLP, methylation-sensitive fragment length polymorphism; GEF, guanine nucleotide exchange factor; COBRA, combined bisulfite and restriction analysis; MSP, methylation-sensitive PCR

Key words: RAPGEF1, C3G, colorectal cancer, squamous cervical cancer, DNA methylation
In contrast, tumor-specific hypomethylation of the gene appears to be frequent in gastrointestinal and gynecological cancers.

\section{Introduction}

Our previous analysis of gastrointestinal cancer by methylationsensitive amplification fragment length polymorphism (MS-AFLP) $(1,2)$ identified several genomic sequences that exhibited methylation alterations in colorectal and gastric cancers (1). One of them (named band CA-8) appeared hypomethylated in colorectal and gastric cancers. Band CA-8 contains a NotI site located in the first intron of RAPGEF1 (GeneID: 2889, chr 9q34.3). This gene functions as a guanine nucleotide exchange factor (GEF) of Rap1 that is involved in multiple effector pathways. Rap1 is known to inhibit MAPK activity by blocking the signal transduction from Ras to c-Raf-1 (3-5), activate the MAPK cascades via B-Raf (6), and activate other members of the MAPK family of proteins (7). Thus, RAPGEF1 can exert pleiotropic effects, promoting or inhibiting growth in different cellular contexts.

The role of RAPGEF1 in cancer progression is unclear. On the one hand, it has been found that overexpression of exogenous RAPGEF1 blocks the anchorage-independent growth induced by cotransfected, activated, sis, ras and v-raf oncogenes in fibroblasts (8); on the other hand, amplification, upregulation and overexpression of endogenous RAPGEF1 have been reported in small cell lung carcinomas, suggesting that this gene can play an oncogenic role through derangement of the CRK-Rap1 signaling pathway (9). More recently, this gene has been reported to undergo silencing through promoter hypermethylation in squamous cervical cancers (10).

We therefore extended the methylation analysis to a panel of squamous cervical tumors to compare the apparent disparity in RAPGEF1 epigenetic alterations in these two types of cancers, i.e., hypermethylation in cervical cancer vs. hypomethylation in colorectal cancer. In contrast with the published data, we found no evidence of hypermethylation in any of the colorectal, cervical or ovarian cancers analyzed, where the gene, 
on the other hand, was found to undergo frequent tumor-specific hypomethylation.

\section{Materials and methods}

Tissue samples. Frozen tissues of gastrointestinal and paired adjacent non-cancerous tissue, and ovarian cancer and normal tissues were obtained from the Cooperative Human Tissue Network (USA). Ovarian tumor samples were analyzed using as control pooled DNA from several normal ovarian tissues. Patients diagnosed to have cervical squamous cell carcinoma were recruited at the Department of Obstetrics and Gynaecology, Prince of Wales Hospital, Hong Kong. Tumor tissues and peripheral blood samples were collected prior to surgical or radiotherapy treatment after obtaining informed consent. Genomic DNA was prepared by standard phenol-chloroform extraction and ethanol precipitation.

Methylation-sensitive amplification length polymorphism. MS-AFLP was performed as described previously (2). Adaptorligated template DNA was PCR amplified with the ${ }^{32} \mathrm{P}$-labeled NotI primer and one of the unlabeled MseI primers. After PCR amplification each PCR sample was electrophoresed on a denaturing gel (Sequagel-6, National Diagnostics, Atlanta, GA) after which the gel was dried and exposed to X-ray film.

COBRA and bisulfite genomic sequencing. For combined bisulfite and restriction analysis (COBRA), genomic DNA was treated with sodium bisulfite using EZ DNA Methylation Kit (Zymo Research, Orange, CA). PCR was performed on the relaxed-criterion CGI located in the first intron of RAPGEF1 (CGI-B) and the strict-criterion CGI located in the promoter region of RAPGEF1 (CGI-A). For COBRA, after amplification, the PCR products were subjected to digestion with the restriction endonuclease BstUI (New England Biolabs, Ipswich, MA), and subsequently resolved on acrylamide gels. For bisulfite genomic sequencing the PCR products were cloned into E. coli DH10B using TOPO-TA cloning Kit (Invitrogen, Carslbad, CA). Plasmids from positive colonies on LB plates containing Kanamycin, XGal and IPTG were prepared using the QIAprep Spin Miniprep kit (Qiagen, Valencia, CA) and inserts checked by electrophoresis after digestion with EcoRI. Plasmids containing the correct-size inserts were subsequently sequenced using M13 reverse primer.

\section{Results}

RAPGEF1 sequences are frequently hypomethylated in colorectal cancers. MS-AFLP is a DNA fingerprinting method that allows identification of sequences containing a NotI site with either of the two CpG sites (GCGGCCGC) hypermethylated or hypomethylated in tumors compared to normal tissue (2). Previous analysis revealed hypomethylation of one of the fingerprint sequences (band CA-8) in $40 \%$ of colorectal and $8 \%$ gastric cancers (1). We subsequently mapped Band CA-8 sequences to chromosome 9q34.3, at the 5' region of RAPGEF1 (Fig. 1). The Not I site detected by MS-AFLP is located in the first intron, $3.85 \mathrm{~kb}$ downstream of its transcription initiation site, in a 615 bp relaxed-criterion $\mathrm{CpG}$ island (CGI-B in Fig. 1).

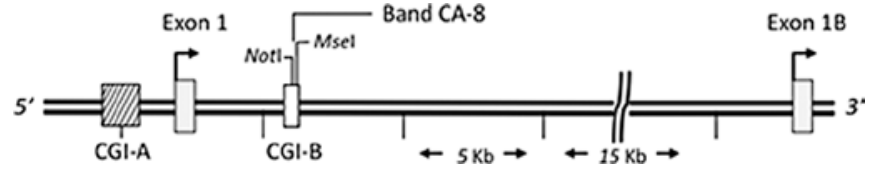

Figure 1. Map of RAPGEF1 5' end region. Band CA-8 is located in a relaxed criterion $\mathrm{CpG}$ island (CpG shore CGI-B, white) in intron 1 on RAPGEF-1. CGI-A is a strict criterion CpG island (CGI-A, stippled) located in the promoter region upstream of exon 1 (grey) of RAPGEF1.

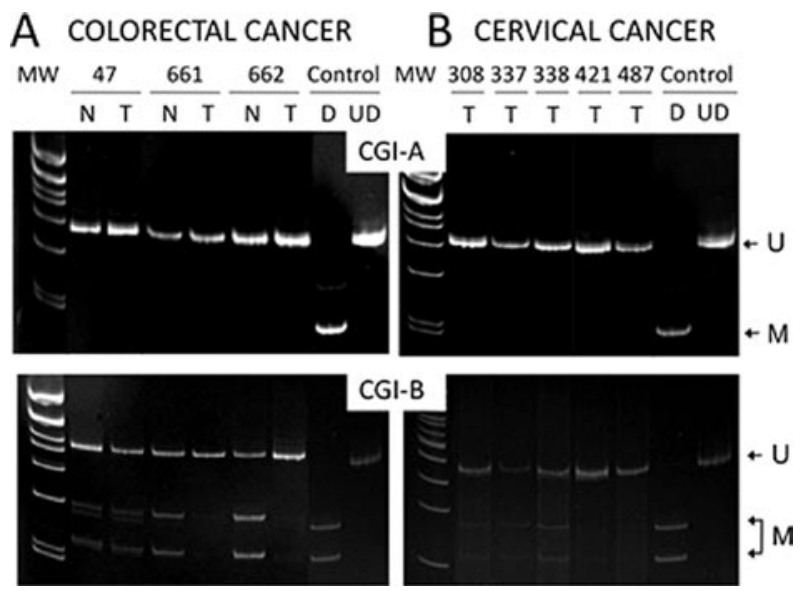

Figure 2. Analysis of the methylation status of CGI-A and CGI-B in RAPGEF1 by COBRA. In COBRA methylation-dependent sequence differences are introduced into the genomic DNA by standard sodium bisulfite treatment and then PCR amplified. In bisulfite treatment unmethylated cytosine residues are converted to uracil and later misread as thymine during PCR amplification, while methylated cytosine residues remain as cytosine. Methylated (M) and unmethylated (U) sequences are distinguished by digestion with restriction endonucleases such as Bst UI (CGCG) that will only cut the methylated sequences where the cytosines are retained. (A) COBRA analysis of CGI-A and CGI-B in three representative colon cancer cases. (B) COBRA analysis of CGI-A and CGI-B in five representative squamous cervical tumor samples. MW, 100 bp molecular weight marker. $\mathrm{N}$ and $\mathrm{T}$, normal and tumor tissue DNA, respectively. D and UD, digested and undigested fully methylated control DNA (New England Biolabs, Ipswich, MA).

To determine the methylation status of this region we analyzed by COBRA 34 matched pairs of colorectal normal and tumor samples that were previously analyzed by MS-AFLP. The results confirmed that this region was mostly methylated in the non-tumor colorectal tissues, but hypomethylated in 13/34 (40\%) of the colorectal tumors (Fig. 2A bottom). To obtain a higher-resolution view of the methylation changes in this region, three cases were further analyzed by bisulfite sequencing. The results confirmed the region was hypomethylated in the tumors relative to normal tissues (Fig. 3).

The RAPGEF1 promoter does not exhibit DNA methylation alterations. Due to the proximity of the hypomethylated CGI-B to a strict-criterion CGI (CGI-A) located in the promoter region of RAPGEF1, we analyzed whether methylation status of the former was related to methylation status of the latter. The results showed no detectable methylation in either normal or tumor tissues (Fig. 2A top).

Methylation status of RAPGEF1 in cervical and ovarian cancers. We further analyzed a 373-bp region within CGI-A covering the $\mathrm{CpG}$ sites previously analyzed by Okino et al, in 


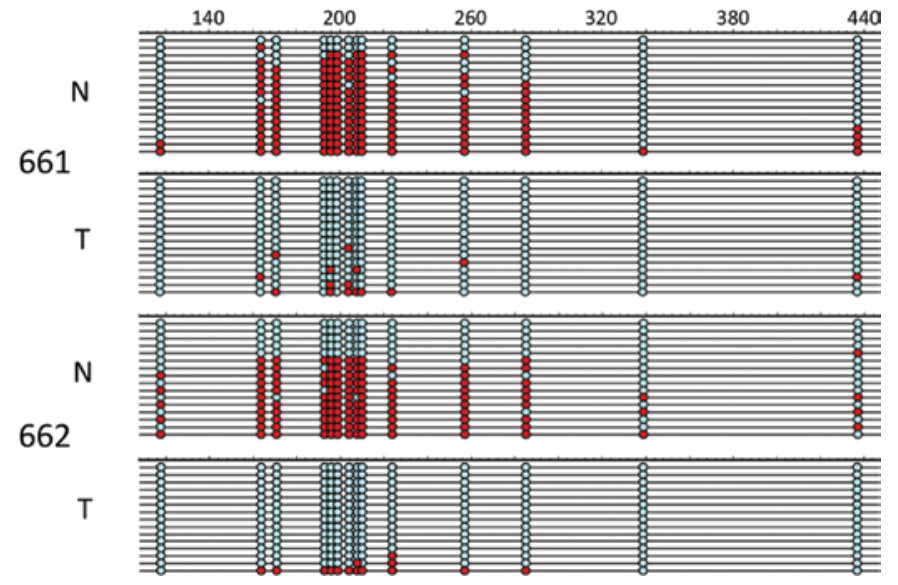

Figure 3. Bisulfite sequencing of CGI-B in colon cancer cases 661 and 662. Each line represents a different cloned DNA fragment. Red circles, methylated CpG sites; blue, unmethylated. $\mathrm{N}$ and $\mathrm{T}$, normal and tumor tissue DNA, respectively. Numbers at top indicate bp relative to the size of the PCR product, starting at the 5 ' end of the forward primer.

cervical squamous carcinomas (10). We found no traces of methylation in any of 29 tumor samples (Fig. 2B top), but found hypomethylation of CGI-B in 8 of 17 (47\%) of the cervical cancers analyzed (Fig. 2B bottom). Analysis of 27 additional ovarian cancers yielded similar results to those of colorectal and cervical cancers: no methylation in CGI-A but hypomethylation in CGI-B in 3/9 endometrial and 6/18 serous cancers $(33 \%)$ relative to normal tissues (data not shown).

\section{Discussion}

Recently, promoter hypermethylation of RAPGEF1 was suggested as a gene silencing mechanism in cervical squamous cell carcinomas (10). A frequency of hypermethylation of $38 \%$ $(5 / 13)$ in cervical tumors of the strict-criterion $\mathrm{CpG}$ island (CGI-A), was reported using methylation-sensitive PCR (MSP) (11). We were thus curious to test the reproducibility of the observation in our larger collection of cervical cancers, because no hypermethylation of this region was found in colorectal cancers. We analyzed CGI-A in cervical and ovarian cancers but we found no evidence of methylation. In addition, we unsuccessfully tried to replicate the results of the experiment described by Okino et al, using the published primers and conditions (10). The reasons for the conflictive results may reside in the design of the PCR primers used in the previous publication. While the reverse primers (both $\mathrm{M}-\mathrm{C} 3 \mathrm{G}-\mathrm{R}$ and UM-C3G-R) correspond to the RAPGEF1 promoter sequence, the forward primers (M-C3G-F and UM-C3G-F) of Okino et al, do not by several base pairs.

The methylation profile of the RAPGEF1 gene in normal colon tissues follows a complex pattern with alternative regions methylated and unmethylated (Fig. 2). We found in colorectal cancer frequent somatic demethylation of one of the gene $\mathrm{CpG}$ rich regions, while the other $\mathrm{CpG}$ island remained unmethylated in both normal and tumor tissues. The somatic demethylation of RAPGEF1 appears to be generalized because similar extent of somatic hypomethylation was found in cervical and ovarian cancers. The demethylation of sequences in cancer, especially of non-CpG islands, is a common observation that in some cases has been associated to the reactivation of the gene normally silenced in normal tissues. Recently it has been reported that in colon cancer methylation alterations occurring in sequences distant from the promoter termed ' $\mathrm{CpG}$ island shores' showed an inverse relationship with gene expression (12).

Our preliminary results did not detect any correlation of gene expression with the methylation status of CGI-B (i.e., re-expression with CGI-B demethylation) in colorectal cancer. However, due to the complex patterns of expression of this gene with alternative splicing affecting different transcripts (13) a more in depth analysis needs to be carried out to determine the potential consequences of demethylation in expression of RAPGEF1.

\section{Acknowledgements}

This work was sponsored by NIH grant R37 CA063585. M.P. is a Research Professor from the Institució Catalana de Recerca i Estudis Avançats (ICREA). We thank Professor Tony Kwok Hung Chung from the Chinese University of Hong Kong, for the helpful contribution of tissues from cervical cancer patients.

\section{References}

1. Suzuki K, Suzuki I, Leodolter A, et al: Global DNA demethylation in gastrointestinal cancer is age dependent and precedes genomic damage. Cancer Cell 9: 199-207, 2006.

2. Yamamoto F, Yamamoto M, Soto JL, et al: Notl-Msell methylation-sensitive amplied fragment length polymorhism for DNA methylation analysis of human cancers. Electrophoresis 22: 1946-1956, 2001.

3. York RD, Yao H, Dillon T, et al: Rap1 mediates sustained MAP kinase activation induced by nerve growth factor. Nature 392: 622-626, 1998.

4. Vossler MR, Yao H, York RD, Pan MG, Rim CS and Stork PJ: cAMP activates MAP kinase and Elk-1 through a B-Raf- and Rap1-dependent pathway. Cell 89: 73-82, 1997.

5. Ohba Y, Mochizuki N, Matsuo K, et al: Rap2 as a slowly responding molecular switch in the Rap1 signaling cascade. Mol Cell Biol 20: 6074-6083, 2000.

6. Ohba Y,Ikuta K, Ogura A, et al: Requirement for C3G-dependent Rap1 activation for cell adhesion and embryogenesis. EMBO J 20: 3333-3341, 2001.

7. Tanaka S, Ouchi T and Hanafusa H: Downstream of Crk adaptor signaling pathway: activation of Jun kinase by v-Crk through the guanine nucleotide exchange protein C3G. Proc Natl Acad Sci USA 94: 2356-2361, 1997.

8. Guerrero C, Martin-Encabo S, Fernandez-Medarde A and Santos E: C3G-mediated suppression of oncogene-induced focus formation in fibroblasts involves inhibition of ERK activation, cyclin A expression and alterations of anchorage-independent growth. Oncogene 23: 4885-4893, 2004.

9. Hirata T, Nagai H, Koizumi K, et al: Amplification, up-regulation and over-expression of $\mathrm{C} 3 \mathrm{G}$ (CRK SH3 domain-binding guanine nucleotide-releasing factor) in non-small cell lung cancers. J Hum Genet 49: 290-295, 2004.

10. Okino K, Nagai $\mathrm{H}$, Nakayama $\mathrm{H}$, et al: Inactivation of $\mathrm{Crk} \mathrm{SH} 3$ domain-binding guanine nucleotide-releasing factor $(\mathrm{C} 3 \mathrm{G})$ in cervical squamous cell carcinoma. Int J Gynecol Cancer 16: 763-771, 2006.

11. Herman JG, Graff JR, Myohanen S, Nelkin BD and Baylin SB: Methylation-specific PCR: a novel PCR assay for methylation status of CpG islands. Proc Natl Acad Sci USA 93: 9821-9826, 1996.

12. Irizarry RA, Ladd-Acosta C, Wen B, et al: The human colon cancer methylome shows similar hypo- and hypermethylation at conserved tissue-specific CpG island shores. Nat Genet 41: 178-186, 2009.

13. Gutierrez-Berzal J, Castellano E, Martin-Encabo S, et al: Characterization of $\mathrm{p} 87 \mathrm{C} 3 \mathrm{G}$, a novel, truncated $\mathrm{C} 3 \mathrm{G}$ isoform that is overexpressed in chronic myeloid leukemia and interacts with Bcr-Abl. Exp Cell Res 312: 938-948, 2006. 